\title{
Pes Anserine Bursitis in Symptomatic Osteoarthritis Patients: A Mesotherapy Treatment Study
}

\author{
Raoul Saggini, MD, PhD, Alexandra Di Stefano, MD, ${ }^{2}$ Ira Dodaj, MD, \\ Laura Scarcello, MD, and Rosa Grazia Bellomo, MD, PhD ${ }^{3}$
}

\begin{abstract}
Background: Pes anserine bursitis strongly affects quality of life in patients with osteoarthritis. Treatment includes nonsteroidal anti-inflammatory drugs (NSAIDs), physiotherapy, and injections of corticosteroid, with highly variable responses; recovery can take 10 days to 36 months. Mesotherapy is a minimally invasive technique consisting of subcutaneous injections of bioactive substances. The goal is to modulate the pharmacokinetics of the injected substance and prolong the effects at a local level.

Objective: To evaluate the effects of mesotherapy with diclofenac for anserine bursitis associated with knee osteoarthritis.

Methods: One hundred and seventeen patients with anserine bursitis associated with grade II Kellgren-Lawrence knee osteoarthritis, assessed by clinical, radiographic, and ultrasonographic examination, were evaluated and treated. They were randomly divided into two groups (A, mesotherapy; B, control). Group A completed nine sessions of mesotherapy with sodium diclofenac $\left(25 \mathrm{mg} / 1 \mathrm{~mL}\right.$; Akis ${ }^{\circledR}$, IBSA, Lugano, Switzerland), $1 \mathrm{~mL}$ for each session, three times per week. Group B received 21 oral administrations of sodium diclofenac $(50 \mathrm{mg}$; Voltaren ${ }^{\circledR}$, Novartis, Parsippany, NJ), once a day for 3 weeks. Primary outcome measures were pain intensity assessed by visual analogue scale (VAS), along with ability to perform activities of daily living, ability to participate in sports, level of pain, symptoms, and quality of life, as assessed by the Knee injury and Osteoarthritis Outcome Score. These measures were performed before and after the treatment period and at 30 and 90 days' follow up.

Results: In both groups pain level decreased significantly after the treatment period. Ultrasonography showed a reduction of the hypoechoic area related to anserine bursitis only in group A.

Conclusion: Administration of conventional NSAIDs (diclofenac) by mesotherapy is effective in managing anserine bursitis in knee osteoarthritis in the short term and mid-term. These observations could be of interest for efforts to reduce the adverse effects of oral administration of anti-inflammatory drugs.
\end{abstract}

\section{Introduction}

D EGENERATIVE OSTEOARTHRITIS (OA) IS the most common arthritic syndrome. The prevalence rate in the general population ranges between $10 \%$ and $12 \%$, making this disease one of the most common causes of pain, activity limitation, and restrictions in participation. Knee osteoarthritis (gonarthrosis) is one of the most frequent forms of degenerative osteoarthritis. In knee osteoarthritis, pain is the primary symptom and probably the most important factor related to activity limitation and disability. ${ }^{1,2}$

Although the pain in knee OA is multifactorial, acute exacerbations are usually closely related to synovial inflammation. Various studies performed by using arthroscopy have shown synovitis in painful knees of patients with knee OA. On the basis of these reports, many investigators focused on noninvasive imaging techniques, such as magnetic resonance imaging and diagnostic ultrasonography. ${ }^{1,3}$

\footnotetext{
${ }^{1}$ Department of Medical Science Oral and Biotechnology, G. D’Annunzio University, Chieti, Italy.

${ }^{2}$ School of Specialties in Physical Medicine and Rehabilitation, G. D'Annunzio University, Chieti, Italy.

${ }^{3}$ Department of Medicine and Science of Aging, G. D'Annunzio University, Chieti, Italy.

(C) Saggini et al., 2015; Published by Mary Ann Liebert, Inc. This Open Access article is distributed under the terms of the Creative Commons Attribution Noncommercial License (http://creativecommons.org/licenses/by-nc/4.0/) which permits any noncommercial use, distribution, and reproduction in any medium, provided the original author(s) and the source are credited.
} 
The term "pes anserinus" refers to the conjoined insertion of the sartorius, gracilis, and semitendinosus muscles along the proximal medial aspect of the tibia. These three muscles are primarily flexors of the knee; they also influence internal rotation of the tibia and protect the knee against rotatory and valgus stress. ${ }^{1-3}$

In pes anserine bursitis, the fluid-filled bursa in the conjoined insertion of the three hamstring muscles becomes inflamed and causes pain. This condition is gradual in onset. It can cause discomfort apart from being responsible for limiting the individual's function. This condition is also known as pes anserinus bursitis or anserine bursitis.,

Patients with this clinical picture may report vague medial knee pain or may present with tenderness and swelling along the proximal medial tibia. Symptoms may be exacerbated when the patient ascends or descends stairs. A diagnosis of pes anserine bursitis should be considered when there is spontaneous pain inferomedial to the knee joint and a history of trauma or diabetes. Conditions associated with pes anserine bursitis include degenerative joint disease of the knee, obesity, valgus knee deformity, pes planus, and sporting activities. ${ }^{4,6-8}$ Pes anserine bursitis affects the quality of life of patients with osteoarthritis, and the treatment methods are different. ${ }^{1,2}$

Treatment includes nonsteroidal anti-inflammatory drugs (NSAIDs), physiotherapy, and injections of corticosteroid, with highly variable responses; recovery can take 10 days to 36 months. ${ }^{9}$ Mesotherapy was introduced 50 years ago by Michel Pistor, a French physician who used this technique as a novel analgesic therapy for a variety of rheumatologic disorders. ${ }^{10,11}$ Mesotherapy is a minimally invasive technique that consists of subcutaneous injections of drugs and, occasionally, plant extracts, homeopathic agents, or other bioactive substances; for this reason, it has been often considered a form of complementary and alternative medicine rather than a conventional medical therapy. ${ }^{11-16}$

The objective of this type of administration is to modulate the pharmacokinetics of the injected substance and to prolong the pharmacologic effects at a local level. One of the main advantages of mesotherapy is that a local pharmacologic effect can be obtained without the need for high systemic concentrations. ${ }^{14,17}$ Intradermal injections of small amounts of active substance where the injection site corresponds to the area of the pathologic condition-for example, in low back pain - may provide clinical benefits when other therapies are not available, are not effective, or cannot be used for whatever reason. ${ }^{17}$ In addition, intradermal administration of active substances in combination with other systemic therapies can produce synergistic effects, and as a result mesotherapy may have dose-sparing effects.

Since its introduction, the use of mesotherapy has been expanded, and therapeutic indications have increased. Although mesotherapy is primarily used for osteoarticular conditions, ${ }^{18-21}$ this technique has recently become popular in cosmetic medicine for treatment of edematous fibrosclerotic panniculopathy and local adiposity. ${ }^{22,23}$

The aim of the current study was to evaluate the effects of mesotherapy with diclofenac for anserine bursitis in knee osteoarthritis.

\section{Materials and Methods}

The multicenter study was conducted at the D'Annunzio University in Chieti, Italy, and at an affiliated rehabilitation center in Florence. It was approved by the local ethics committee and was performed in accordance with the 1964 Declaration of Helsinki.

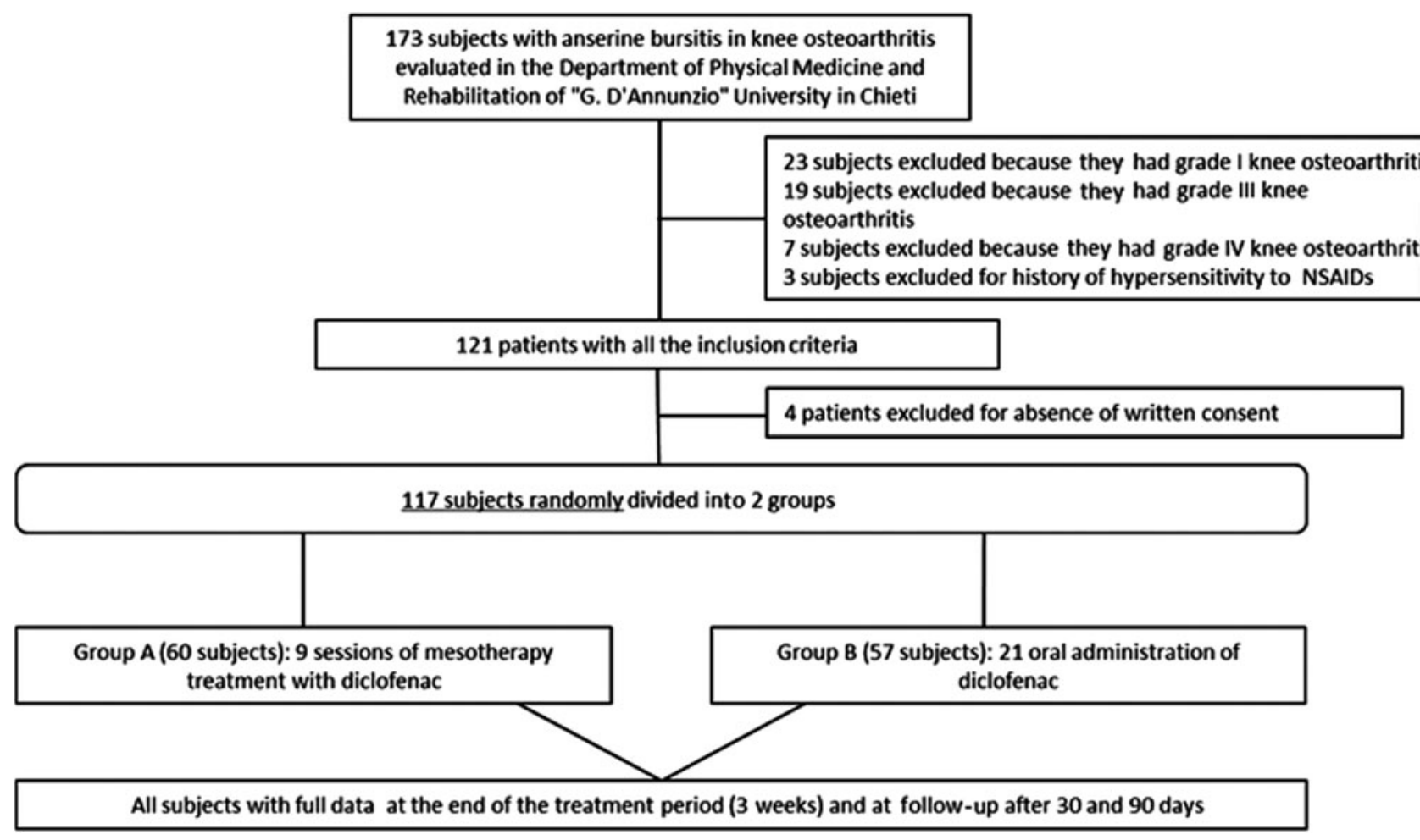

FIG. 1. Study flowchart. NSAIDs, nonsteroidal anti-inflammatory drugs. 
One hundred and seventeen patients (59 men and 58 women) age 18-56 years (average age, 36 years) were evaluated and treated. All them had anserine bursitis, ascertained clinically and by ultrasonography, in association with grade II Kellgren-Lawrence knee osteoarthritis (Fig. 1). Inclusion criteria were age between 10 and 60 years, grade II Kellgren-Lawrence knee osteoarthritis, re-acutization of pain, and synovial inflammation. Exclusion criteria were pregnancy, known hypersensitivity to products, infiltrative therapy with hyaluronic acid, polynucleotides or corticosteroids in progress, drug abuse or alcohol abuse, significant comorbidities (such as the presence of neurologic abnormalities, concomitant severe rheumatic disease, and systemic abnormalities, such as diabetes), a surgical intervention within 3 months before the study, psychiatric conditions, or psychotherapy or physical therapy within 5 weeks before the study.

During the study period, patients in the mesotherapy were not allowed to take corticosteroids or NSAIDs. None of the enrolled patients received any other medical intervention during the study.

The patients were randomly divided into two groups (A, mesotherapy; B, control) after giving written consent and agreeing to the possible treatment (local or oral).

Group A (60 patients; 30 men and 30 women) received nine sessions of mesotherapy with sodium diclofenac (25 mg/1 mL; Akis®, IBSA, Lugano, Switzerland), $1 \mathrm{~mL}$ for each session, three times per week. Group B (57 patients; 29 men and 28 women) received 21 oral administrations of sodium diclofenac (50 mg; Voltaren $\AA$, Novartis, Parsippany, NJ), once every day for 3 weeks.

All patients reported pain during sleep and after a long period of rest, as well as reduction of pain after about a 200$\mathrm{m}$ walk. All patients were asked to provide standard radiographs (anterior-posterior and lateral) of the lumbosacral tract and knee joints.

Primary outcome measures were pain intensity, quantified by using a $10-\mathrm{cm}$ millimetric visual analogue scale (VAS), on which the patient is asked to mark the degree of pain intensity, ranging from 0 (absence of pain) to 10 (the worst pain imaginable), along with abilities in activities of daily living (ADLs), ability to participate in sports, level of pain, symptoms, and quality of life, as assessed by the Knee injury and Osteoarthritis Outcome Score (KOOS). All these assessments, along with ultrasonography, were performed before (T0) and after (T1) the treatment period and at 30 days' (T2) and 90 days' (T3) follow-up in all patients.

All data are given as means \pm standard deviations. Differences between mean values before and after the rehabilitation period were tested for significance by using two-way analysis of variance for repeated measures. The minimum level of statistical significance was set at $p<0.05$. GraphPad Prism (version 6) software (Abacus Concepts GraphPad Software, San Diego, CA).

\section{Results}

In group $\mathrm{A}$, the subjective pain levels assessed by VAS were $7 \pm 1.05$ at $\mathrm{T} 0,2 \pm 0.35$ at $\mathrm{T} 1,3.8 \pm 0.85$ at $\mathrm{T} 2$, and $4.0 \pm 0.85$ at T3 (Fig. 2). The KOOS findings were as follows. Disability during sports activity at T1 decreased significantly

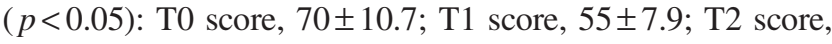
$60 \pm 8.5$; T3 score, $60 \pm 8.7$ (Fig. 3). Perceived symptoms also

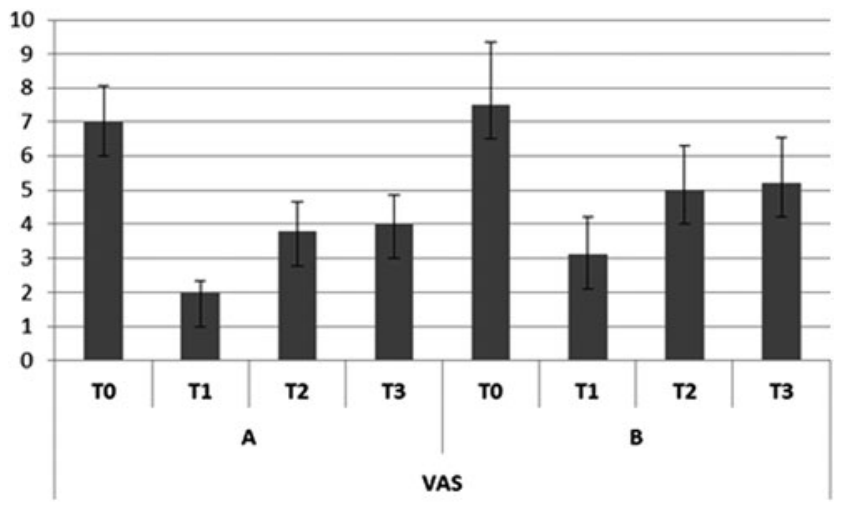

FIG. 2. Trend of subjective pain assessed by visual analogue scale (VAS). Error bars represent the standard deviation. A, Group A (mesotherapy treatment); B, Group B (oral treatment).

were significantly reduced $(p<0.05)$ at $\mathrm{T} 1$ and at both fol-

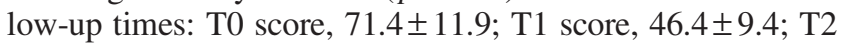

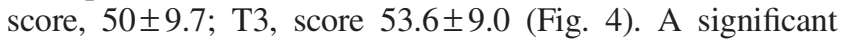
reduction $(p<0.05)$ was seen in the effect of osteoarthritis on quality of life at $\mathrm{T} 1$ and at both follow-up times: T0 score,

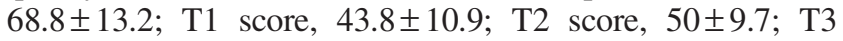
score, $50 \pm 9.8$ (Fig. 5). Disability with regard to ADLs also decreased significantly $(p<0.05)$ at T1 and at both follow-up

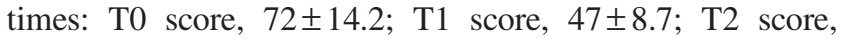
$47 \pm 8.7$; T3 score, $50 \pm 9.2$ (Fig. 6). Finally, pain perception was significantly reduced $(p<0.01)$ at $\mathrm{T} 1$ and at both followup times: T0 score, $75 \pm 10.3$; T1 score, $30.5 \pm 5.5$; T2 score, 36 \pm 5.2 ; T3 score, $41 \pm 5.8$ (Fig. 7).

In group $\mathrm{B}$, the subjective pain levels assessed by VAS were $7.5 \pm 1.85$ at $\mathrm{T} 0,3 \pm 1.10$ at $\mathrm{T} 1$, and 5 at $\mathrm{T} 2$ and $\mathrm{T} 3$ (Fig. 2). The KOOS findings were as follows: Disability during sports activity did not significantly decrease: T0 score, $75 \pm 15.8$; T1 score, $65 \pm 15.5$; T2 score, $65 \pm 15.7$; T3 score, $70 \pm 17.2$ (Fig. 3). A significant reduction occurred $(p<0.05)$ in perceived symptoms at T1: T0 score, $71.4 \pm 13.1$; T1 score, $60.7 \pm 8.3$; T2 score, $64.3 \pm 8.7$; T3 score, $64.3 \pm 9.1$ (Fig. 4). The effect of osteoarthritis on quality of life decreased significantly $(p<0.05)$ at T1: T0 score, 75 \pm 13.2 ; T1 score, $50 \pm 11.0$; T2 score, 68.8 \pm 14.5 ; T3 score, 68.8 \pm 14.7 (Fig. 5). Disability with regard to ADLs was reduced significantly $(p<0.05)$ at T1: T0 score,

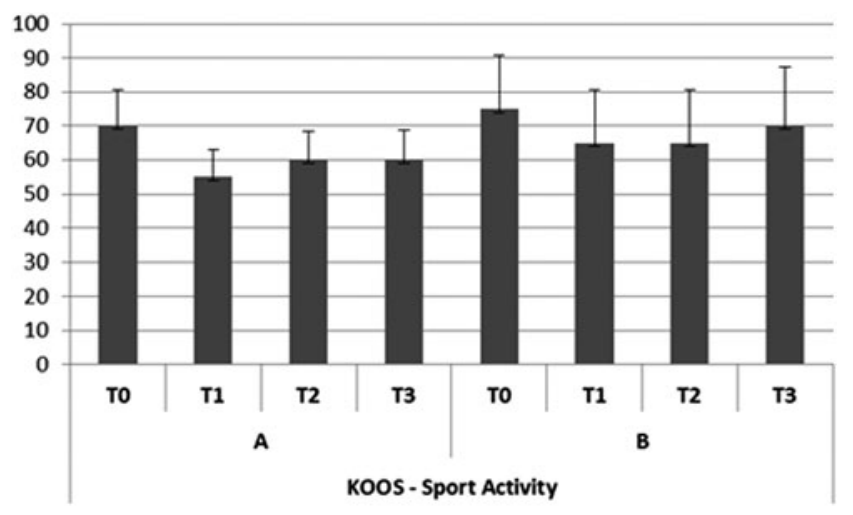

FIG. 3. Trend of disability during sports activity (Knee injury and Osteoarthritis Outcome Score [KOOS]). 


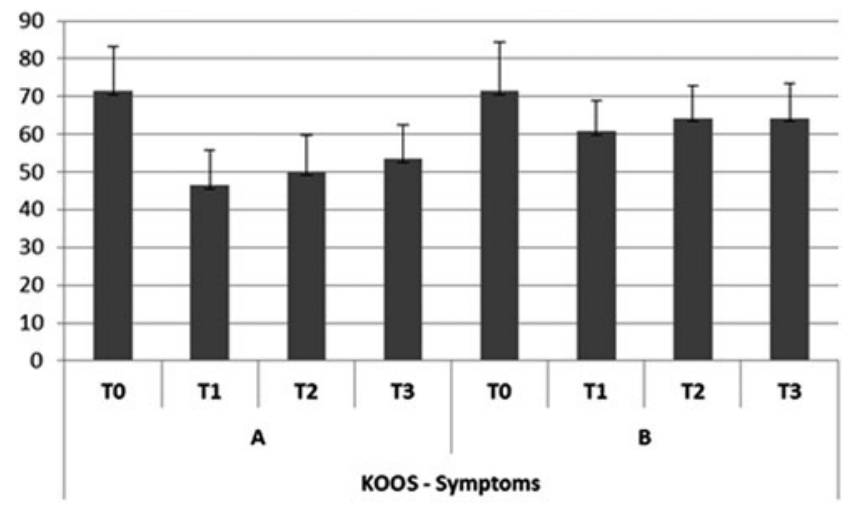

FIG. 4. Trend of perceived symptoms (KOOS).

70.6 \pm 12.7 ; T1 score, $61.7 \pm 9.4$; T2 score, $66.2 \pm 10.9$; T3 score, $67.6 \pm 12.5$ (Fig. 6). Finally, a significant reduction $(p<0.05)$ was seen in pain perception at $\mathrm{T} 1$ and at both follow-up times: T0 score, $75 \pm 10.4$; T1 score, 52.7 \pm 8.3 ; T2 score, 55.6 \pm 8.8 ; T3 score, $58.3 \pm 9.5$ (Fig. 7).

In both groups, pain level and absence of pain at nighttime decreased significantly after the treatment period (T1). In particular, group A had pain remission after a 120-m walk at T1 and after a 160-m walk at T2 and T3. Ultrasonography showed a reduction of the hypoechoic area related to anserine bursitis only in group A (ie, the mesotherapy group).

\section{Discussion}

The aim of this study was to evaluate the effectiveness of anti-inflammatory drugs administered via mesotherapy in patients with local inflammation in grade II Kellgren-Lawrence knee osteoarthritis. Present results showed that the administration of NSAIDs (diclofenac) via mesotherapy can provide the same therapeutic benefit as that induced by conventional (oral) drug administration in relation to pain after a 3-week treatment period. Indeed, both treatments significantly reduced pain intensity and disability in daily life activity; the effect was maintained up to 3 months (30 days' and 90 days' follow-up) only in the group that received mesotherapy. These results are in accordance with previous studies showing that naproxen and diclofenac administered via mesotherapy were more effective than those given orally. ${ }^{20,24,25}$

The major finding of this study is the similar effectiveness of mesotherapy and conventional systemic therapy, despite

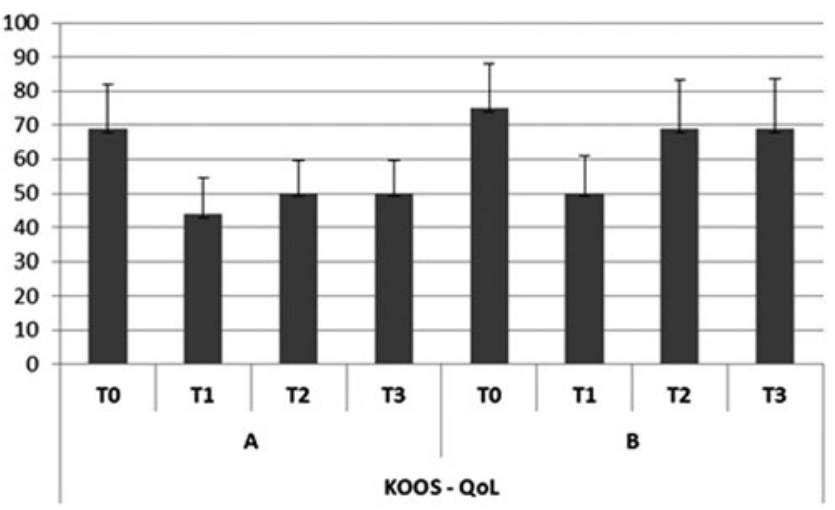

FIG. 5. Effect of osteoarthritis on quality of life (QoL) (KOOS).

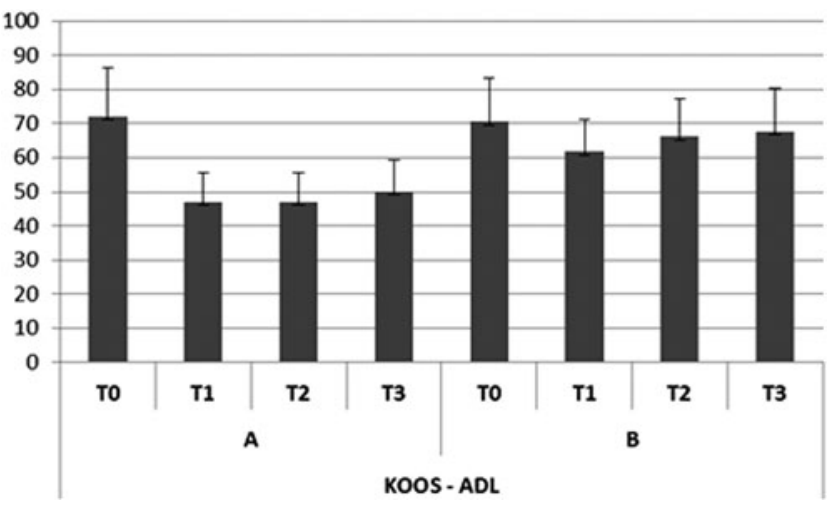

FIG. 6. Trend of disabilities with regard to activities of daily living (ADLs) (KOOS).

the lower amount of drugs administered to patients undergoing mesotherapy ( $25 \mathrm{mg}$ with mesotherapy vs $50 \mathrm{mg}$ with oral administration) and the lower short-term risk for adverse reactions in the mesotherapy group (3 weeks).

The similar efficacy of mesotherapy and conventional therapy, despite different drug dosages, is difficult to explain. Subcutaneous drug administration results in a very slow drug absorption in comparison with other systemic routes, such as oral and intramuscular. Thus, it could be hypothesized that anti-inflammatory drugs administered via mesotherapy achieve a higher drug concentration in the subcutaneous tissue and exert local effects close to inflammatory cells, sensory fibers, and vascular mediators that orchestrate inflammation and pain. This hypothesis could be confirmed by the evidence of reduction of the anserine bursitis showed by ultrasonography in group A (the mesotherapy group) but not in group B (the oral administration group).

Although this study did not measure drug plasma levels after the two routes of administration, it is possible to hypothesize that mesotherapy resulted in a lower systemic bioavailability of drugs, with consequent lower incidence of adverse reactions. This could offer a great therapeutic advantage given the high rates of adverse effects associated with NSAID or corticosteroid use in the elderly population. ${ }^{10,26-28}$

Although mesotherapeutic techniques used in dermatologic surgery have been associated with many adverse effects at injection sites, including atypical mycobacterial

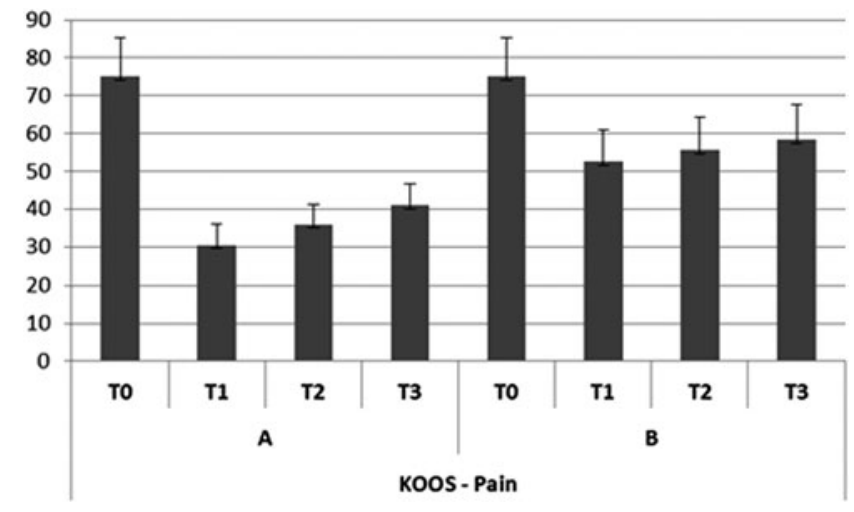

FIG. 7. Trend of subjective pain (KOOS). 
infections, urticaria, lichenoid drug eruptions, and psoriasis, no evidence of local reactions was found in the present study. ${ }^{29}$

In conclusion, results of this study indicate that combined administration of conventional NSAIDs (diclofenac) by mesotherapy is an effective and well-tolerated method for managing anserine bursitis in II grade knee osteoarthritis in the short term and mid-term compared with oral administration of the same drug with different concentrations. A possible limitation of the study is the absence of drug plasma levels. However, future studies could have a larger scale and thus also measure plasma bioavailability of the drug. These observations could be of potential interest for the pharmacologic treatment of anserine bursitis in order to reduce the adverse effects associated with high plasma levels of anti-inflammatory drugs.

\section{Author Disclosure Statement}

No competing financial interests exist.

\section{References}

1. Eşen S, Akarırmak U, Aydın FY, Unalan H. Clinical evaluation during the acute exacerbation of knee osteoarthritis: the impact of diagnostic ultrasonography. Rheumatol Int 2013;33:711-717.

2. Ayral X, Pickering EH, Woodworth TG, Mackillop N, Dougados M. Synovitis: a potential predictive factor of structural progression of medial tibiofemoral knee osteoarthritis: results of a 1 year longitudinal arthroscopic study in 422 patients. Osteo Arthr Cartil 2005;13:361-367.

3. Walther M, Harms H, Krenn V, Radke S, Faehndrich TP, Gohlke F. Correlation of power Doppler sonography with vascularity of the synovial tissue of the knee joint in patients with osteoarthritis and rheumatoid arthritis. Arthr Rheum 2001;44:331-338.

4. Uysal F, Akbal A, Gökmen F, Adam G, Reşorlu M. Prevalence of pes anserine bursitis in symptomatic osteoarthritis patients: an ultrasonographic prospective study. Clin Rheumatol 2015;34:529-533.

5. Gnanadesigan N, Smith RL. Knee pain: osteoarthritis or anserine bursitis? J Am Med Dir Assoc 2003;4:164-166.

6. Wood LR, Peat G, Thomas E, Duncan R. The contribution of selected non-articular conditions to knee pain severity and associated disability in older adults. Osteoarthr Cartil 2008;16:647-653.

7. Imani F, Rahimzadeh P, Abolhasan Gharehdag F, Faiz SH Sonoanatomic variation of pes anserine bursa. Korean J Pain 2013;26:249-254.

8. Alvarez-Nemegyei J. Risk factors for pes anserinus tendinitis/bursitis syndrome: a case control study. J Clin Rheumatol 2007;13:63-65.

9. Helfenstein M Jr, Kuromoto J. Anserine syndrome. Rev Bras Reumatol. 2010;50:313-327.

10. Costantino C, Marangio E, Coruzzi G. Mesotherapy versus systemic therapy in the treatment of acute low back pain: a randomized trial. Evid Based Complement Alternat Med. 2011; pii: 317183.

11. Pistor M. What is mesotherapy? Chir Dent Fr 1976;46:59-60.

12. Dalloz-Bourguignon A. A new therapy against pain: mesotherapy. J Belge Med Phys Rehabil 1979;2:230-234.
13. de Beir J, Bazon H. On the subject of mesotherapy. Chir Dent Fr 1984;54:27-28.

14. Mammucari M, Gatti A, Maggiori S, Sabato AF. Role of mesotherapy in musculoskeletal pain: opinions from the Italian Society of Mesotherapy. Evid Based Complement Alternat Med 2012; 436959.

15. Rohrich RJ. Mesotherapy: what is it? Does it work? Plast Reconstr Surg. 2005;15;115:1425.

16. Maggiori S. Manuale di Intradermoterapia Distrettuale. La Mesoterapia in Italia, EMSI, Roma, Italy, 2004.

17. Mammucari M, Gatti A, Maggiori S, Bartoletti CA, Sabato AF. Mesotherapy, definition, rationale and clinical role: a consensus report from the Italian society of Mesotherapy. Eur Rev Med Pharmacol Sci 2011;6:682-694.

18. Pitzurra M, Cavallo R, Farinelli S, et al. On the intradermal inoculation of antibiotics: some experimental data. Giornale di Mesoterapia 1981;1:9-14.

19. Pitzurra M, Marconi P. Immunogenesis and mesotherapy: the immunoresponse to antigens inoculated intradermally. Giornale di Mesoterapia 1981;1:9-14.

20. Coudeville L, Andre P, Bailleux F, Weber F, Plotkin S. A new approach to estimate vaccine efficacy based on immunogenicity data applied to influenza vaccines administered by the intradermal or intramuscular routes. Human Vaccines 2010;6:841-848.

21. Hsu CC, Kuo HC, Hsu CT, Gu Q. Abdominal mesotherapy injection extended the absorption of follicle-stimulating hormone. Fertil Steril 2011;95:2134-2136.

22. Crenna P, Mancia P. Reflex actions in mesotherapy. Giornale di Mesoterapia 1981;1:29-40.

23. Sticchi L, Alberti M, Alicino C, Crovari P. The intradermal vaccination: past experiences and current perspectives. J Prev Med Hygiene 2010; 51:7-14.

24. Colombo I, Cigolini M, Combi F. Clinical results of the multicentric experimentation. Giornale di Mesoterapia 1981; $1: 50-52$.

25. Saraceni V, Palieri G, De Pedis M. Clinical results of the multicentric experimentation. Giornale di Mesoterapia 1981; 1:53-59.

26. Sostres C, Gargallo CJ, Arroyo MT, Lanas A. Adverse effects of non-steroidal anti-inflammatory drugs (NSAIDs, aspirin and coxibs) on upper gastrointestinal tract. Best Pract Res Clin Gastroenterol 2010;24:121-132.

27. Whittle BJR. Gastrointestinal effects of non steroidal antiinflammatory drugs. Fundament Clin Pharmacol 2003; 17:301-313.

28. Schacke H, Docke WD, Asadullah K. Mechanisms involved in the side effects of glucocorticoids. Pharmacol Therap 2002;96:23-43.

29. Konda D, Thappa DM. Mesotherapy: What is new? Indian J Dermatol Venereol Leprol 2013;79:127-134.

Address correspondence to: Raoul Saggini, $M D, P h D$

Department of Medical Science Oral and Biotechnology G. D’Annunzio University Viale Abruzzo 322 Chieti, Abruzzo 66100 Italy

E-mail: saggini@unich.it 